\title{
ON INTERPOLATION POLYNOMIALS OF THE HERMITE-FEJÉR TYPE II
}

\author{
S.J. Goodenough and T.M. Mills
}

Given a real-valued function $f$ on $[-1,1], n \in N$, and the following partition of $[-1,1]$ :

$$
-1<x_{n}<x_{n-1}<\ldots<x_{k}:=\cos ((2 k-1) \pi / 2 n)<\ldots<x_{1}<1 \text {, }
$$

there exists a unique polynomial $R_{4 n-1}(f ; x)$ of degree not exceeding $4 n-1$ such that

$$
R_{4 n-1}\left(f ; x_{k}\right)=f\left(x_{k}\right) \text { for } k=1,2,3, \ldots, n
$$

and, for $j=1,2$ and 3 ,

$$
R_{4 n-1}^{(j)}\left(f ; x_{k}\right)=0 \text { for } k=1,2,3, \ldots, n \text {. }
$$

The polynomials $R_{4 n-1}(f ; x)$ for $n=1,2,3, \ldots$ are called Hermite-Fejer type interpolation polynomials based on the zeros of $T_{n}(x):=\cos \left(n \cos ^{-1} x\right)$.

In this article, the authors first outline the development of results pertaining to the (rate of) convergence of Hermite-Fejer type interpolation polynomials. They then extend this development by deriving a new pointwise error estimate. This estimate represents a marked improvement on all previous error estimates in that it reflects the interpolation conditions.

Received 3 November 1980. The authors wish to express their appreciation to Miss R. Myors and Miss L. Henderson for their expert assistance in the preparation of this paper. 


\section{Introduction}

Let $f$ be a real-valued function defined on $[-1,1]$ and, for $k=1,2,3, \ldots, n$, denote by

$$
x_{k}=\cos ((2 k-1) \pi / 2 n)
$$

the zeros of the Chebyshev polynomial of the first kind

$$
T_{n}(x)=\cos n \theta \text { where }-1 \leq x=\cos \theta \leq 1 \text {. }
$$

Then there exists a unique polynomial $R_{4 n-1}(f ; x)$ of degree at most $4 n-1$ satisfying the following conditions:

$$
R_{4 n-1}\left(f ; x_{k}\right)=f\left(x_{k}\right) \text { for } k=1,2,3, \ldots, n
$$

and, for $j=1,2$ and 3 ,

$$
R_{4 n-1}^{(j)}\left(f ; x_{k}\right)=0 \text { for } k=1,2,3, \ldots, n \text {. }
$$

Henceforth, we shall refer to the polynomials $R_{4 n-1}(f ; x)$ as Hermite-Fejér type interpolation polynomials. These are so named due to their close connection with the Hermite-Fejér interpolation polynomials used by Fejer [1] in 1916 in presenting a new proof of the celebrated Weierstrass approximation theorem.

Indeed, the Hermite-Fejér type interpolation polynomials were first introduced by Krylov and Stevermann [5] (in 1922), who stated the following

THEOREM 1 [Krylov and Steuermann]. If $f \in C[-1,1]$, then

$$
\lim _{n \rightarrow \infty}\left\|R_{4 n-1}(f)-f\right\|_{\infty}=0 \text {. }
$$

where $\|\cdot\|_{\infty}$ denotes the uniform norm on $\mathrm{C}[-1,1]$.

Unfortunately, the proof given by Krylov and Steuermann was incorrect. A correct proof of this result was later furnished by Laden [6]. In 1959, Stancu [10] estimated the rate of convergence of the Hermite-Fejer type polynomials $R_{4 n-1}(f ; x), n=1,2,3, \ldots$ in terms of the modulus of continuity of $f$, denoted by $w(f ; \delta)$, where

$$
w(f ; \delta)=\sup \{|f(x)-f(y)|:|x-y| \leq \delta, x, y \in[-1,1]\} .
$$

THEOREM 2 [Stancu]. There is a constant $c_{1}>0$ such that, if 
$f \in C[-1,1]$, then

$$
\left\|R_{4 n-1}(f)-f\right\|_{\infty} \leq C_{1} w(f ; 1 / \sqrt{n}) \text { for } n=1,2,3, \ldots \text {. }
$$

Stancu's result was subsequently improved by florica [2], who proved the following

THEOREM 3 [Florica]. There is a constant $c_{2}>0$ such that, if $f \in C[-1,1]$, then

$$
\left\|R_{4 n-1}(f)-f\right\|_{\infty} \leq c_{2} w(f ;(\log n) / n) \text { for } n=2,3,4, \ldots
$$

Now suppose $\Omega$ is an increasing, subadditive, continuous function defined on $[0, \infty[$ with $\Omega(0)=0$. Indeed, it may be helpful to think of $\Omega$ as a generalised modulus of continuity. Furthermore, for each fixed but arbitrary positive constant $M$, put

$$
C_{M}(\Omega):=\{f \in C[-1,1]: w(f ; \delta) \leq M \Omega(\delta) \text { for all } \delta \geq 0\} \text {. }
$$

One of the authors, Mills [7], focussed attention on $C_{1}(\Omega)$ to obtain an estimate (for the error incurred in replacing $f$ by $R_{4 n-1}(f)$ ) which is best possible in a certain sense.

THEOREM 4 [Mills]. There exist positive constants $C_{3}$ and $C_{4}$ such that

$$
\begin{aligned}
c_{3} / n \sum_{k=2}^{n} \Omega(1 / k) \leq \sup _{f \in C_{1}(\Omega)}\left\|R_{4 n-1}(f)-f\right\|_{\infty} \leq c_{4} / n \cdot \sum_{k=1}^{n} \Omega(1 / k) & \\
& \text { for } n=2,3,4, \ldots .
\end{aligned}
$$

In 1978, Prasad [8] established the following more general pointwise estimate.

THEOREM 5 [Prasad]. There is a positive constant $C_{5}$ such that, if $f \in C_{M}(\Omega), n=2,3,4, \ldots$ and $x \in[-1,1]$,

$$
\left|R_{4 n-1}(f ; x)-f(x)\right| \leq C_{5} M / n \sum_{k=1}^{n} \Omega\left(\left(\sqrt{1-x^{2}} / k\right)+\left(1 / k^{2}\right)\right) .
$$

It should be mentioned that this result was observed independently, but not proved, by Goodenough and Mills [3]. The main purpose of this 
paper is to establish

THEOREM 6. There exist positive constants $c_{1}$ and $c_{2}$ such that, if $f \in C[-1,1], n=2,3,4, \ldots$ and $x \in[-1,1]$,

(5) $\left|R_{4 n-1}(f ; x)-f(x)\right| \leq\left(c_{1} T_{n}(x)^{4} / n\right) \sum_{k=1}^{n} w\left(f ;\left(\sqrt{1-x^{2}} / k\right)+\left(1 / k^{2}\right)\right)$

$$
+c_{2} w\left(f ;\left(\left|T_{n}(x)\right| / n\right)\right) \text {. }
$$

Note that the above theorem represents an improvement on Theorem 5 in that it reflects the interpolation conditions. More precisely, if $x=x_{k}=\cos ((2 k-1) \pi / 2 n)$, then both sides of inequality (5) vanish simultaneously.

\section{Technical preliminaries}

In this section, we shall unveil certain technical results which are needed for the proof of Theorem 6. Firstly, the formula for the HermiteFejér type interpolation polynomial $R_{4 n-1}(f ; x)$ is given by:

$$
R_{4 n-1}(f ; x)=\sum_{k=1}^{n} f\left(x_{k}\right) s_{k}(x)
$$

where

$$
\begin{aligned}
& S_{k}(x)=F_{k}(x)+G_{k}(x)+H_{k}(x), \\
& F_{k}(x)=\left(1 / n^{4}\right)\left(1-x_{k}^{2}\right)\left(1-x^{2}\right)\left(T_{n}(x) /\left(x-x_{k}\right)\right)^{4}, \\
& G_{k}(x)=\left(\left(4 n^{2}-1\right) / 6 n^{4}\right)\left(x-x_{k}\right)^{2}\left(1-x_{k}\right)\left(T_{n}(x) /\left(x-x_{k}\right)\right)^{4}, \\
& H_{k}(x)=\left(1 / 2 n^{4}\right)\left(T_{n}(x)^{2} /\left(x-x_{k}\right)\right)^{2}
\end{aligned}
$$

and

$$
x_{k}=\cos ((2 k-1) \pi / 2 n) \text { for } k=1,2,3, \ldots, n \text {. }
$$

We have already reported that if $x=x_{k}$ for some $k=1,2,3, \ldots, n$, then both sides of the inequality (5) vanish simultaneously. Accordingly, we may suppose that $x \neq x_{k}$ for 
$k=1,2,3, \ldots, n$. In this case, we define $x_{j}$ to be the node of interpolation which is closest to $x$ : if there are two such nodes, then choose either one (but not both) to be $x_{j}$. Clearly $j=j(n)$, and provided $x \neq 1, j+\infty$ as $n \rightarrow \infty$.

The following lemma is due to Kis ([4], p. 30).

LEMMA 1 [Kis]. For $-1 \leq x=\cos \theta \leq 1$,

$$
\left|f\left(x_{k}\right)-f(x)\right| \leq\left\{\begin{array}{l}
2 w(f ;(\sin \theta) / n)+2 w\left(f ; 1 / n^{2}\right) \text { if } k=j, \\
5 w(f ;(i \sin \theta) / n)+13 w\left(f ; i^{2} / n^{2}\right) \text { if } i=|k-j| \geq 1 .
\end{array}\right.
$$

The next lemma will be needed to establish the term $C_{2} w\left(f ;\left|T_{n}(x)\right| / n\right)$ which appears as part of the upper estimate for $\left|R_{4 n-1}(f ; x)-f(x)\right|$ in Theorem 6 .

LEMMA 2. If $x=\cos \theta, x_{k}=\cos \theta_{k}$ for $k=1,2, \ldots, n$ and $x_{j}$ is the node closest to $x$, then $\left|\theta-\theta_{j}\right| \leq(\pi / 2 n)|\cos n \theta|$.

Proof. Suppose for example that $\theta_{j}<\theta \leq\left(\theta_{j}+\theta_{j+1}\right) / 2$. Then the establishment of the required inequality follows from the easy observation that the absolute value of the gradient of the line joining $\left(\theta_{j}, 0\right)$ and $\left(\left(\theta_{j}+\theta_{j+1}\right) / 2, \cos n\left(\theta_{j}+\theta_{j+1}\right) / 2\right)$ does not exceed the absolute value of the gradient of the line joining $\left(\theta_{j}, 0\right)$ and $(\theta, \cos n \theta)$. Other cases may be treated in a similar vein.

It is debatable whether the following lemma deserves its title. Nonetheless, the elementary inequalities contained therein will smooth the way for proving the all-important Lemme 4 .

LEMMA 3. If $\alpha, \beta \in[0, \pi]$, then

(i) $0 \leq \sin \alpha \leq 2 \sin \frac{1}{2}(\alpha+\beta)$, and

(ii) $\sin \frac{1}{2}(\alpha+\beta) \geq \sin \frac{3}{2}|\alpha-\beta|$.

LEMMA 4. For $k=1,2, \ldots, j-1, j+1, \ldots, n, F_{k}(x), G_{k}(x)$ and 
$H_{k}(x)$ are all expressible in the form $O(1) T_{n}(x)^{4} / i^{2}$, where $i=|k-j|$.

Proof. Now

$$
\begin{aligned}
F_{k}(x) & =\left(1 / n^{4}\right)\left(1-x_{k}^{2}\right)\left(1-x^{2}\right)\left[T_{n}(x) /\left(x-x_{k}\right)\right]^{4} \\
& =\left(T_{n}(x)^{4} / n^{4}\right) \cdot\left(\sin ^{2} \theta_{k} /\left(4 \sin ^{2}\left(\left(\theta+\theta_{k}\right) / 2\right)\right)\right) \cdot\left(\sin ^{2} \theta /\left(4 \sin ^{2}\left(\left(\theta+\theta_{k}\right) / 2\right)\right)\right) \\
& \quad \cdot\left(1 /\left(\sin ^{4}\left(\left(\theta-\theta_{k}\right) / 2\right)\right)\right) \\
& \leq\left(T_{n}(x)^{4} / n^{4}\right) \cdot\left(1 /\left(\sin ^{4}\left(\left(\theta-\theta_{k}\right) / 2\right)\right)\right) \quad \text { by Lemma } 3 .
\end{aligned}
$$

But

(11) $\left[1 /\left(\sin ^{4}\left(\left(\theta-\theta_{k}\right) / 2\right)\right)\right]=O(1) /\left(\theta-\theta_{k}\right)^{4}=O(1) n^{4} / i^{4}$, where $i=|k-j|$ so it follows that

$$
F_{k}(x)=O(1) T_{n}(x)^{4} / i^{4}
$$

In particular,

$$
F_{k}(x)=O(1) T_{n}(x)^{4} / i^{2}
$$

Secondly,

$$
\begin{aligned}
G_{k}(x)= & \left(\left(4 n^{2}-1\right) / 6 n^{4}\right) \cdot\left(x-x_{k}\right)^{2}\left(1-x x_{k}\right)\left(T_{n}(x) /\left(x-x_{k}\right)\right)^{4} \\
< & \left(2 T_{n}(x)^{4} / 3 n^{2}\right) \cdot\left(\left(1-x_{k}^{2}+x_{k}^{2}-x x_{k}\right) /\left(x-x_{k}\right)^{2}\right) \\
= & \left(2 T_{n}(x)^{4} / 3 n^{2}\right) \cdot\left(\sin ^{2} \theta_{k} /\left(4 \sin ^{2}\left(\left(\theta+\theta_{k}\right) / 2\right)\right)\right) \cdot\left(1 /\left(\sin ^{2}\left(\left(\theta-\theta_{k}\right) / 2\right)\right)\right) \\
& +\left(T_{n}(x)^{4} / 3 n^{2}\right) \cdot\left(\left(\cos \theta_{k}\right) /\left(\sin \left(\left(\theta+\theta_{k}\right) / 2\right) \sin \left(\left|\theta-\theta_{k}\right| / 2\right)\right)\right) \\
\leq & \left(2 T_{n}(x)^{4} / 3 n^{2}\right) \cdot\left(1 /\left(\sin ^{2}\left(\left(\theta-\theta_{k}\right) / 2\right)\right)\right) \\
\leq & \left.+\left(2 T_{n}(x)^{4} c_{1} / 3 i^{2}\right)+\left(T_{n}\right)^{4} / 3 n^{2}\right) \cdot\left(1 /{ }^{4} c_{1} / 3 i^{2}\right) \text { for some absolute constant } c_{1} .
\end{aligned}
$$

Thus

$$
G_{k}(x)=o(1) T_{n}(x)^{4} / i^{2}
$$


Finally,

$$
\begin{aligned}
H_{k}(x) & =\left(1 / 2 n^{4}\right)\left[T_{n}(x)^{2} /\left(x-x_{k}\right)\right]^{2} \\
& =\left(T_{n}(x)^{4} / 2 n^{4}\right) \cdot\left(1 /\left(4 \sin ^{2}\left(\left(\theta+\theta_{k}\right) / 2\right) \sin ^{2}\left(\left(\theta-\theta_{k}\right) / 2\right)\right)\right) \\
& \leq\left(T_{n}(x)^{4} / 8 n^{4}\right) \cdot\left(1 /\left(\sin ^{4}\left(\left(\theta-\theta_{k}\right) / 2\right)\right)\right) \text { by Lemma } 3 .
\end{aligned}
$$

Thus

$$
H_{k}(x)=O(1) T_{n}(x)^{4} / i^{4} \text { by }(11)
$$

In particular,

$$
H_{k}(x)=O(1) T_{n}(x)^{4} / i^{2}
$$

and the lemma is proved.

We now have the necessary machinery for the

\section{Proof of Theorem 6}

From the definition of $R_{4 n-1}(f ; x)$, we have

$$
R_{4 n-1}(1 ; x)=\sum_{k=1}^{n} S_{k}(x) \equiv 1 .
$$

It follows that

$$
\text { (12) } \begin{aligned}
& \left|R_{4 n-1}(f ; x)-f(x)\right| \\
= & \left|\sum_{k=1}^{n}\right| f\left(x_{k}\right)-f(x)\left|s_{k}(x)\right| \\
\leq & \sum_{k=1}^{n}\left|f\left(x_{k}\right)-f(x)\right| s_{k}(x) \\
= & \sum_{k=1}^{j-1}\left|f\left(x_{k}\right)-f(x)\right| s_{k}(x)+\left|f\left(x_{j}\right)-f(x)\right| s_{j}(x)+\sum_{k=j+1}^{n}\left|f\left(x_{k}\right)-f(x)\right| s_{k}(x) \\
= & W_{1}+w_{2}+w_{3}, \text { say. }
\end{aligned}
$$

We now estimate $W_{1}, W_{2}$ and $W_{3}$ in turn. 
Firstly,

$$
\begin{aligned}
W_{1} & =\sum_{k=1}^{j-1}\left|f\left(x_{k}\right)-f(x)\right| s_{k}(x) \\
& =O(1) T_{n}(x)^{4} \sum_{k=1}^{j-1}\left(1 / i^{2}\right)\left[5 w(f ;(i \sin \theta) / n)+13 w\left(f ; i^{2} / n^{2}\right)\right] \\
& =o(1)\left(T_{n}(x)^{4} / n\right) \sum_{k=1}^{n} w\left(f ;\left(\sqrt{1-x^{2}} / k\right)+\left(1 / k^{2}\right)\right)
\end{aligned}
$$

Similarly we may show that

$$
W_{3}=O(1)\left(T_{n}(x)^{4} / n\right) \sum_{k=1}^{n} w\left(f ;\left(\sqrt{1-x^{2}} / k\right)+\left(1 / k^{2}\right)\right)
$$

It remains only to estimate $W_{2} \cdot$ Now

$$
\begin{aligned}
W_{2} & =\left|f\left(x_{j}\right)-f(x)\right| s_{j}(x) \\
& \leq\left|f\left(x_{j}\right)-f(x)\right| \\
& \leq w\left(f ;\left|x_{j}-x\right|\right) \\
& \leq w\left(f ;\left|\theta_{j}-\theta\right|\right) \\
& \leq 2 v\left(f ;\left|T_{n}(x)\right| / n\right) \text { by Lemma } 2 .
\end{aligned}
$$

We conclude that (12), (13), (14) and (15) prove Theorem 6 .

\section{References}

[1] Leopold Fejér, "Ueber Interpolation", Nachr. Ges. Wissench. Göttingen (1916), 66-91.

[2] Olariu Florica, "Asupra ordinului de aproximatie prin polinoame de interpolare de tip Hermite-Fejér cu noduri cvadruple" [On the order of approximation by interpolating polynomials of HermiteFejér type with quadruple nodes], An. Univ. Timisoara Ser. Sti. Mat.-Fiz. 3 (1965), 227-234.

[3] S.J. Goodenough and T.M. Mills, "The asymptotic behaviour of certain interpolation polynomials", J. Approx. Theory (to appear). 
[4] 0. Ниш [0. Kis], "Замечання о порядне сходимости лаграннева интерполирования" [Remark on the order of convergence of Lagrange interpolation], Ann. Univ. Sci. Budapest. Eötvös Sect. Math. 11 (1968), 27-40.

[5] N.M. Krylov and E. Steuermann, "Sur quelques formulae d'interpolation. convergentes pour toute fonction continue", BuZl. Acad. de Z'oucraine 1 (1923), 13-16.

[6] H.N. Laden, "An application of the classical orthogonal polynomials to the theory of interpolation", Duke Math. J. 8 (1941), 591-610.

[7] T.M. Mills, "On interpolation polynomials of the Hermite-Fejér type", Colloq. Math. 35 (1976), 159-163.

[8] J. Prasad, "On the rate of convergence of interpolation polynomials of Hermite-Fejér type", Bull. Austral. Math. Soc. 19 (1978), 29-37.

[9] R.B. Saxena, "A note on the rate of convergence of Hermite-Fejér interpolation polynomials", Canad. Math. Bull. 17 (1974), 299-301.

[10] D.D. Stancu, "Asupra unei demonstratii a teoremei Iui Weierstrass" [On a proof of the theorem of Weierstrass], Bul. Inst. Politehn. Iagi (N.S.) 5 (9) (1959), 47-50.

Department of Mathematics,

Bendigo College of Advanced Education,

Bendigo,

Victoria 3550,

Australia. 